\title{
Diagnostic performance of an algorithm for automated collateral scoring on computed tomography angiography
}

\author{
Lennard Wolff $^{1}$ (D) Simone M. Uniken Venema ${ }^{2}$ - Sven P. R. Luijten ${ }^{1} \cdot$ Jeannette Hofmeijer ${ }^{3}$. Jasper M. Martens ${ }^{4}$. \\ Marie Louise E. Bernsen ${ }^{4}$ - Adriaan C. G. M. van Es ${ }^{5}$ - Pieter Jan van Doormaal ${ }^{6}$ - Diederik W. J. Dippel ${ }^{6}$.

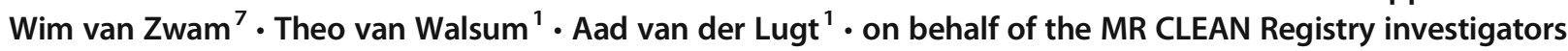

Received: 11 October 2021 / Revised: 24 December 2021 / Accepted: 29 January 2022 / Published online: 4 March 2022

(C) The Author(s) 2022

\begin{abstract}
Objectives Outcome of endovascular treatment in acute ischemic stroke patients depends on collateral circulation to provide blood supply to the ischemic territory. We evaluated the performance of a commercially available algorithm for assessing the collateral score (CS) in acute ischemic stroke patients.

Methods Retrospectively, baseline CTA scans ( $\leq 3$-mm slice thickness) with an intracranial carotid artery (ICA), middle cerebral artery segment M1 or M2 occlusion, from the MR CLEAN Registry $(n=1627)$ were evaluated. All CTA scans were evaluated for visual CS (0-3) by eight expert radiologists (reference standard). A Web-based AI algorithm quantified the collateral circulation $(0-100 \%)$ for correctly detected occlusion sides. Agreement between visual CS and categorized automated CS (0: $0 \%, 1:>0-\leq 50 \%, 2:>50-<100 \%, 3: 100 \%$ ) was assessed. Area under the curve (AUC) values for classifying patients in having good (CS: 2-3) versus poor (CS: 0-1) collaterals and for predicting functional independence (90-day modified Rankin Scale 0-2) were computed. Influence of CTA acquisition timing after contrast material administration was reported.

Results In the analyzed scans $(n=1024), 59 \%$ agreement was found between visual CS and automated CS. An AUC of 0.87 (95\% CI: 0.85-0.90) was found for discriminating good versus poor CS. Timing of CTA acquisition did not influence discriminatory performance. AUC for predicting functional independence was 0.66 (95\% CI 0.62-0.69) for automated CS, similar to visual CS 0.64 (95\% CI 0.61-0.68).

Conclusions The automated CS performs similar to radiologists in determining a good versus poor collateral score and predicting functional independence in acute ischemic stroke patients with a large vessel occlusion.

Key Points

- Software for automated quantification of intracerebral collateral circulation on computed tomography angiography performs similar to expert radiologists in determining a good versus poor collateral score.

- Software for automated quantification of intracerebral collateral circulation on computed tomography angiography performs similar to expert radiologists in predicting functional independence in acute ischemic stroke patients with a large vessel occlusion.

- The timing of computed tomography angiography acquisition after contrast material administration did not influence the performance of automated quantification of the collateral status.
\end{abstract}

Keywords Ischemic stroke $\cdot$ Collateral circulation $\cdot$ Algorithms

Lennard Wolff

1.wolff.1@erasmusmc.nl

1 Department of Radiology and Nuclear Medicine, Erasmus MC University Medical Center, Rotterdam, The Netherlands

2 Department of Neurology and Neurosurgery, University Medical Center Utrecht, Utrecht, The Netherlands

3 Department of Neurology, Rijnstate Hospital, Arnhem, The Netherlands
4 Department of Radiology, Rijnstate Hospital, Arnhem, The Netherlands

5 Department of Radiology, Leiden University Medical Center, Leiden, The Netherlands

6 Department of Neurology, Erasmus MC University Medical Center, Rotterdam, The Netherlands

7 Department of Radiology and Nuclear Medicine, Maastricht University Medical Center, Maastricht, The Netherlands 


\begin{tabular}{ll}
\multicolumn{2}{l}{ Abbreviations } \\
AUC & Area under the curve \\
CI & Confidence interval \\
CS & Collateral score \\
EVT & Endovascular treatment \\
LVO & Large vessel occlusions \\
MCA & Middle cerebral arteries \\
MIP & Maximum intensity projection \\
qCS & Quantitative automated collateral score \\
vCS & Visual collateral score
\end{tabular}

\section{Introduction}

Endovascular treatment (EVT) is the preferred option for patients with ischemic stroke due to large intracranial vessel occlusion [1]. Clinical outcome after EVT is affected by patient characteristics, workflow parameters, and imaging-derived parameters [2]. An important imaging-based parameter which predicts outcome is the extent of collateral circulation to provide blood supply to the ischemic territory [3]. This can be used to select patients who may benefit from endovascular treatment [4]. Conventional collateral scoring systems use a categorical scale, which is based on assessing visual differences in vascular filling of the middle cerebral arteries (MCA) regions between the affected and unaffected hemispheres [5]. However, visual collateral scores (vCS) assessed by a radiologist or stroke physician have substantial inter- and intra-observer variations [6].

Fast, reliable, and consistent automated scoring of the collaterals could not only reduce time delay before initiating treatment but also reduce human error in judgment, which may improve treatment outcome. Moreover, since the qCS is a quantitative measure, it might give more information on the degree of collateral circulation than the visual categorical collateral score. Several automated image algorithms to assess collateral status in acute ischemic stroke patients have been developed [7-11]. The aim of this study was to evaluate the performance of a commercially available algorithm for assessing the collateral score in baseline CTA images of acute ischemic stroke patients.

\section{Methods}

\section{Study population}

We used data from the Multicenter Randomized Clinical Trial of Endovascular Treatment of Acute Ischemic Stroke Registry (MR CLEAN Registry) in The Netherlands, which collects consecutive patient data from all stroke intervention centres that perform endovascular treatment (EVT) [12]. Collateral score or the severity and the extent of early ischemic changes were not used as exclusion criteria for EVT. Detailed MR CLEAN Registry eligibility criteria were published previously [12].
The study protocol was evaluated by the Erasmus Medical Center ethics committee (Rotterdam, the Netherlands), who granted permission to carry out the study as a registry (MEC-2014-235). We selected all MR CLEAN Registry patients with a large vessel occlusion of the anterior circulation undergoing EVT from March 16, 2014, until June 15, 2016 $(n=1627)$. All patients with available baseline source CTA data were eligible for inclusion. Imaging parameters which needed to be met to process the CTA scan by StrokeViewer included axial series with a slice thickness $\leq 3 \mathrm{~mm}$; slice increment equal to or smaller than slice thickness; minimum matrix size of $512 \times 512$; full head coverage; detection of affected cerebral hemisphere by the algorithm. Additional inclusion and exclusion criteria are shown in Fig. 1. This study was performed in accordance with the STARD guidelines for reporting diagnostic accuracy [13].

\section{Imaging core lab assessments}

All baseline CTA scans were evaluated for a 4-point collateral score by a core lab of eight expert radiologists $(5-20$ years of experience). The collateral score indicates the percentage of collateral supply in the occluded vessel territory in comparison to the contralateral side (0: absent, $1:>0 \%$ and $\leq 50 \%, 2$ : $>50 \%$ and $<100 \%, 3: 100 \%$ ), as shown in Fig. 2 [5]. Occlusion location was also assessed and subdivided in ICA, and M1 and M2 segments. The expert readers were

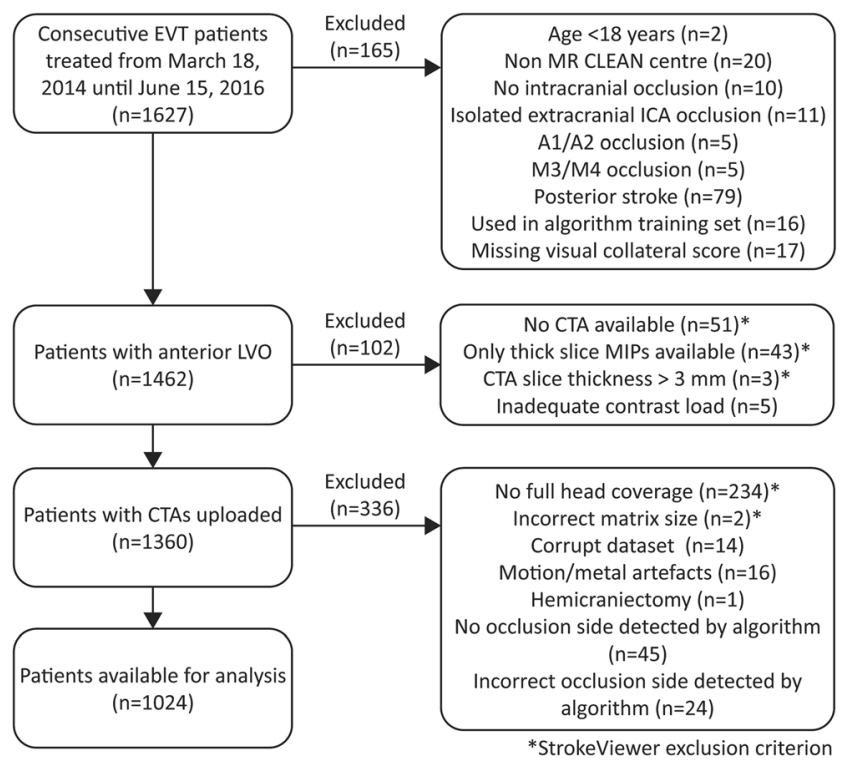

Fig. 1 Patient selection. *StrokeViewer exclusion criterion. EVT, endovascular treatment; CTA, computed tomography angiography; LVO, large vessel occlusion; ICA, internal carotid artery; ICA, internal carotid artery terminus; M1, M1 segment of the middle cerebral artery; M2, M2 segment of the middle cerebral artery; A1/A2, A1 or A2 segment of the anterior cerebral artery; M3/M4, M3 or M4 segment of the middle cerebral artery; MIP, maximum intensity projection; $\mathrm{mm}$, millimeters 


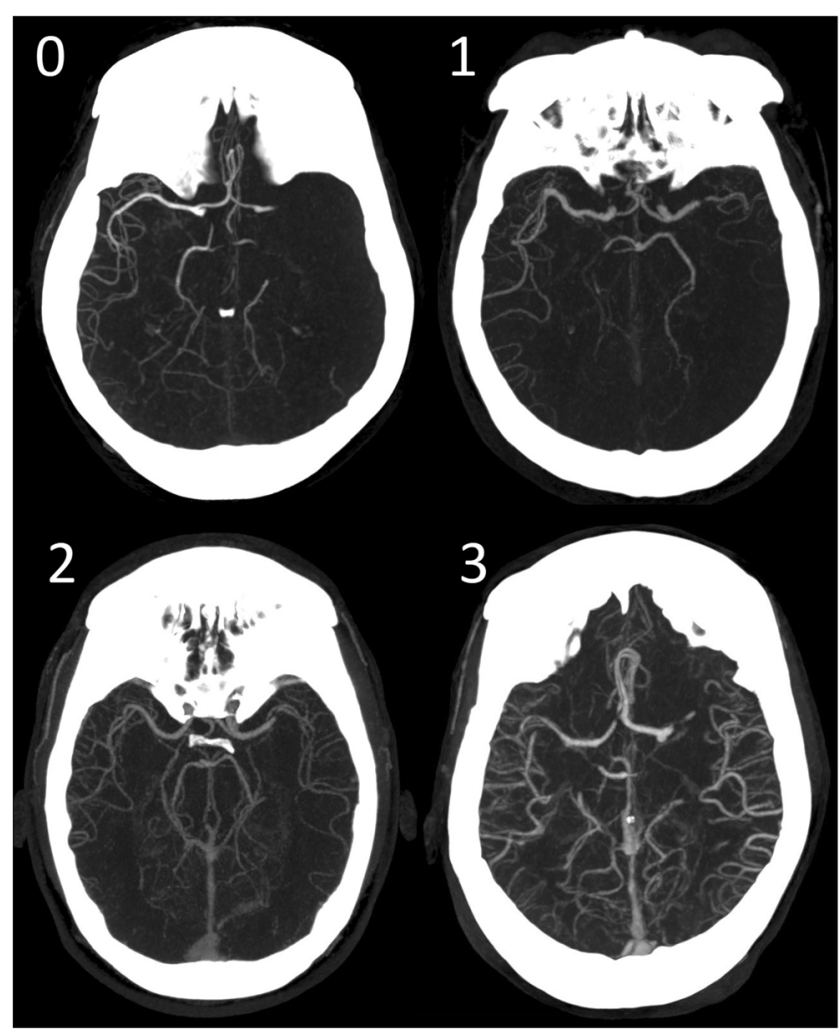

Fig. 2 Visual collateral score grading in patients with an M1 occlusion. 0: absent collaterals, $0 \%$ filling of the occluded territory. 1: poor collaterals, $>0 \%$ and $\leq 50 \%$ filling of the occluded territory. 2 : moderate collaterals, $>50 \%$ and $<100 \%$ filling of the occluded territory. 3: good collaterals, $100 \%$ filling of the occluded territory

blinded for automated collateral score and all clinical information except for symptomatic cerebral hemisphere.

\section{Automated collateral score workflow}

StrokeViewer (v2.1.22, Nicolab B.V.) uses a Web-based AI algorithm based on a deep learning convolutional neural network to automatically identify large vessel occlusion location combined with automated vessel segmentations to produce automated collateral scores. StrokeViewer is hosted on the Google Cloud Platform (Google LLC). A previously published quantitative method for collateral scoring has been used as basis for the development of the current algorithm [14]. First, the DICOM requirements are checked (e.g., image modality, slice thickness, image dimensions). Second, all CTA scan slices without brain tissue are removed. Then, a brain segmentation algorithm is applied to create a brain mask. The resulting image is aligned to a reference brain atlas.

To compute a collateral score, first, the hemisphere with ischemic stroke is identified. StrokeViewer includes an occlusion detection algorithm for LVO in the anterior circulation. The occlusion location is indicated with a cube on the processed CTA scan. The occlusion detection algorithm performance is described elsewhere [15]. Once the occlusion location is detected, the collateral score is computed (quantitative scale, $0-100 \%$ ) for both the specific area affected by the occlusion, defined as the area with detected vessels distal from the found occlusion, and for the whole MCA region. A score of $0 \%$ corresponds to no vascular filling in the assessed region, whereas $100 \%$ reflects non-impaired vascular filling in the assessed region. CTA scan phase (early arterial, peak arterial, equilibrium, early venous, late venous) was also detected automatically by StrokeViewer to determine the reliability of the collateral score [16].

A visual presentation of the occlusion location and the MCA vessel trees segmentation is generated and stored in DICOM format, which is accessible for the clinician. In addition, PDF reports with CTA maximum intensity projection (MIP) snapshots showing the occlusion location and MCA vessel tree segmentations, the collateral score, and other findings are generated. StrokeViewer produced results but showed a warning when scan quality deemed to be insufficient (early arterial, late venous or unknown scan phase, slice thickness > $2.0 \mathrm{~mm}$ ). All information is accessible in StrokeViewer. The clinician is notified by email once the results are generated. Since we did not develop StrokeViewer, more algorithm details were not available to us.

\section{Automated collateral score assessments}

CTA scan processing time was defined as the time between the finished CTA series upload and the receipt of a notification that the results are generated. StrokeViewer was used to access the generated PDF reports with the algorithm results for each patient. The results were subsequently stored in a database for further analysis.

\section{Statistical analysis}

All scans with a visual and automated collateral score were analyzed. Analysis was done with the quantitative automated collateral score (qCS) for the affected region, unless stated otherwise. From all scans included in the analyses, a random sample of 200 CTA scans was scored by two expert radiologists to provide interobserver agreements as percentage and as intraclass correlation coefficient (ICC) using a two-way random-effects, absolute agreement, single-rater/measurement model (ICC [2,1]). We presented the distribution of qCS per vCS (0-3). To assess collateral score (CS) agreement between the software and expert readers, the qCS was categorized in 4 groups $(0: 0 \%, 1:>0-\leq 50 \%, 2:>50-\leq 100,3: 100 \%)$. Agreement between the qCS by the algorithm and the vCS by expert readers was calculated for all CS categories $(0-3)$ and for dichotomized vCS categories (poor: 0-1, good: 2-3). Results were displayed in an error matrix. Scans with a difference $>1$ between the $\mathrm{vCS}$ and categorized qCS were reviewed and rescored by the first and last author independently (L.W., A.L.) and findings were reported. Agreement was also assessed 
with the ICC using a one-way random-effects, absolute agreement, single-rater/measurement model (ICC $[1,1])$. The strictest ICC model was used because a selection of readers out of a panel of multiple expert readers assessed the collateral score [17]. For the ICC, values less than 0.5 are indicative of poor reliability, values between 0.5 and 0.75 indicate moderate reliability, values between 0.75 and 0.9 indicate good reliability, and values greater than 0.90 indicate excellent reliability.

The dichotomized vCS was used to calculate the area under receiver operating characteristic curves (AUC) for the qCS of the affected region and the qCS of the whole MCA region for all occlusion locations combined and for the different occlusion locations (ICA, M1 and M2 segments). Next, qCS performance was computed for sufficient-quality scans and insufficientquality scan categories (according to StrokeViewer) separately.

Finally, AUCs were computed for predicting functional independence (90-day modified Rankin Scale $0-2$ ) with vCS (0-3), categorized qCS (0-3), and qCS (continuous) of the affected region and qCS (continuous) of the whole MCA region. Differences in AUC were evaluated using the method of DeLong [18]. When appropriate, 95\% confidence intervals (CI) were reported. Statistical analyses were performed using the SPSS software package (version 25.0.0.1) and R statistical software (version 4.0.4) with R package pROC.

\section{Results}

Data from 1024 patients were available for analysis (Fig. 1). CTA scans were acquired using 28 different scanner types from all major vendors (Philips, Siemens, Toshiba, Canon Medical, GE Healthcare) in $>50$ hospitals. Mean processing time from finished upload to qCS result was $5 \mathrm{~min}(\mathrm{SD} \pm 72$ seconds). Baseline characteristics are reported in the supplementary materials, table 1 .
For the vCS, we found an interobserver agreement of $65 \%$ and an ICC of 0.66 (95\% CI: $0.55-0.75)$ between two expert radiologists in a random sample of 200 analyzed CTA scans. A review of the disconcordant cases with a difference of $>1$ between the vCS and categorized qCS $(n=37)$ showed a difference of $0(n=22)$ or $1(n=9)$ with qCS, or an incorrect vessel segmentation by StrokeViewer $(n=6)$. The distribution of qCS per vCS (0-3) is displayed in a boxplot (Fig. 3). After categorizing the $\mathrm{qCS}$ in collateral score $0-3$ and comparing it to the visual CS, we found 59\% agreement, $37 \%$ disagreement with a score difference of $1,3 \%$ disagreement with a score difference of 2 , and $1 \%$ disagreement with a score difference of 3 (Table 1). CS dichotomization (poor: $0-1$, good: $2-3$ ) resulted in an agreement of $81 \%$ (Table 1). Comparing the categorized qCS $(0-4)$ to the visual $\mathrm{CS}$ resulted in a moderate ICC of 0.60 (95\% CI: $0.55-0.62$ ), which increased to 0.61 (95\% CI: 0.57-0.65) after CS dichotomization.

After dichotomizing the vCS in poor and good collaterals, overall AUC was 0.87 (95\% CI: 0.85-0.90) for discrimination with automated collateral score (Table 2). When using the whole MCA region as target downstream territory, in comparison to using the region affected by the found occlusion, the performance was significantly lower $(p<0.01)$. For patients with an ICA occlusion (AUC: 0.89 ; 95\% CI $0.85-0.93$ ) and M1 occlusion (AUC: $0.88 ; 95 \%$ CI $0.85-0.91$ ) the performance was better than for M2 occlusions (AUC: 0.77; 95\% CI 0.68-0.86) (Table 2).

Of the analyzed patients, $40 \%(n=1024)$ had a CTA scan with insufficient quality according to StrokeViewer. Compared to sufficient-quality CTA scans, qCS performance was similar for all CTA scans with insufficient quality: early arterial phase scans $(n=246, p=0.62)$, late venous phase scans $(n=72, p=0.09)$, and scans with a slice thickness $>2.0 \mathrm{~mm}(n=111, p=0.96)$. Since qCS performance was not influenced by appointed scan quality, we included and analyzed all patients $(n=1024)$ (Table 3$)$.

Table 1 Error matrix for visual collateral score vs. automated collateral score

\section{Automated collateral score}

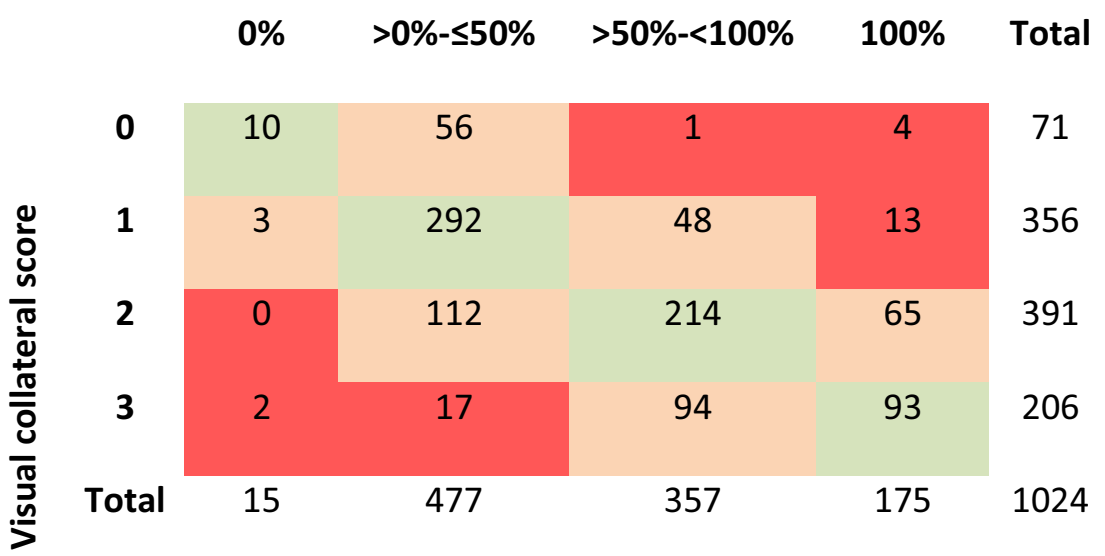




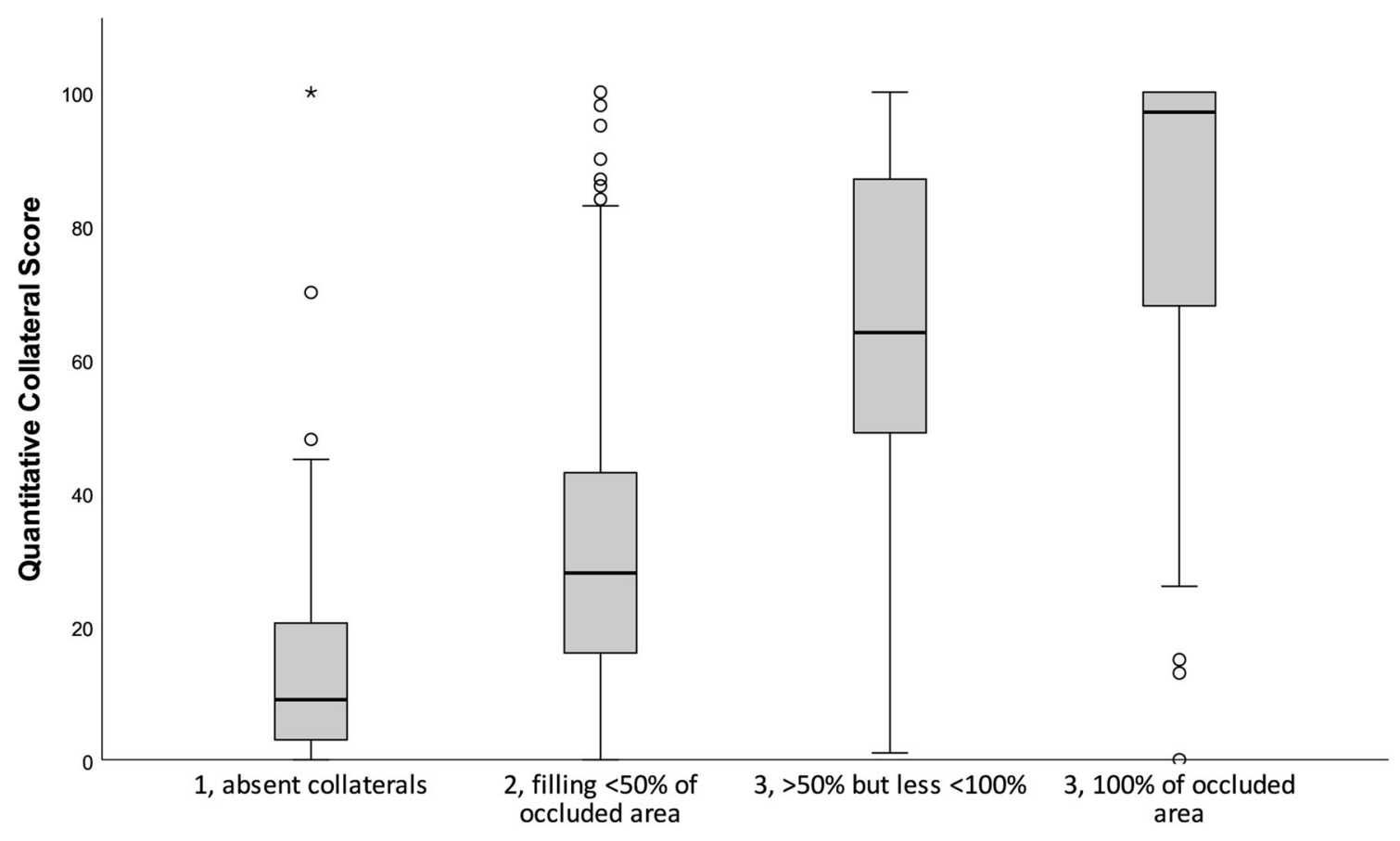

Visual Collateral Score

Fig. 3 Quantitative collateral score distribution for visual collateral score categories. Distribution of quantitative collateral scores per visual collateral score. Box plots with median, interquartile range, and 95\% confidence intervals. Circle: one patient. Asterisk: four patients

The AUC for predicting functional independence (90-day modified Rankin Scale 0-2) with the vCS was 0.64 (95\% CI: $0.61-0.68)$ and this increased to 0.66 (95\% CI $0.62-0.69)$ when using qCS (Table 4). The AUC remained unchanged when using the whole MCA region instead of the occluded MCA region to compute the qCS (Table 4). AUC decreased to $0.63(95 \%$ CI $0.60-0.67)$ after qCS categorization in 4 groups (0: $0 \%, 1:>0-\leq 50 \%, 2:>50-<100 \%, 3: 100 \%)$, but in comparison to the vCS, this difference was not statistically significant $(p=0.29)$ (Table 4).

\section{Discussion}

In this study, we analyzed the performance of automated collateral scoring on baseline CTA scans in 1024 acute ischemic stroke patients with a large vessel occlusion in the MCA territory.
This study demonstrated an agreement of 59\% between the categorized automated collateral score and the visual collateral score, which increased to $81 \%$ after classifying patients in having good (CS: $2-3)$ or poor (CS: $0-1)$ collaterals. This is comparable to interrater agreements in this study and previous studies $[4,6,8]$. The qCS performance for M2 occlusions was lower than that for M1 and ICA, which could be explained by misclassifications of $\mathrm{M} 2$ occlusions by the thrombus detection algorithm [15]. The timing of CTA acquisition after administering intravenous contrast did not influence quantitative CS performance. The accuracy in predicting functional independence (90-day mRS 0-2) was similar for automated and visual collateral scoring.

Several studies have been published on automation of the CS with comparable performance when compared to visual scoring. A previous study on 442 patients reported the results of the same collateral score algorithm and compared it to the

Table 2 ROC curve characteristics for predicting dichotomized visual collateral score using the quantitative collateral score

\begin{tabular}{lllrr}
\hline Occlusion location $(\%)$ & $n$ & Affected MCA region & Whole MCA region & $\begin{array}{l}\text { AUC difference* } \\
\text { AUC (95\% CI) }\end{array}$ \\
\hline All (100) & 1024 & $0.87(0.85-0.90)$ & $0.85(0.82-0.87)$ & $<0.01$ \\
ICA (24.9) & 255 & $0.89(0.85-0.93)$ & $0.89(0.85-0.93)$ & 0.67 \\
M1 (62.8) & 643 & $0.88(0.85-0.91)$ & $0.84(0.81-0.88)$ & $<0.01$ \\
M2 (12.3) & 126 & $0.77(0.68-0.86)$ & $0.76(0.67-0.85)$ & 0.52 \\
\hline
\end{tabular}

Dependent variable: dichotomized visual collateral score (0-1: poor collaterals, 2-3: good collaterals). $M C A$, middle cerebral arteries; $A U C$, Area Under the Curve; $I C A$, internal carotid artery terminus; $M 1$, M1 segment of the middle cerebral artery; $M 2, \mathrm{M} 2$ segment of the middle cerebral artery. *Using the Delong test 
Table 3 ROC curve characteristics for predicting dichotomized visual collateral score using the quantitative collateral score

\begin{tabular}{lll}
\hline Scan phase $^{\mathrm{a}}(\%)$ & $n$ & $\begin{array}{l}\text { Occluded MCA region } \\
\text { AUC }(95 \% \text { CI) }\end{array}$ \\
\hline All (100) & 1024 & $0.87(0.85-0.90)$ \\
Early arterial (24.0) & 246 & $0.86(0.81-0.91)$ \\
Peak arterial (25.4) & 260 & $0.92(0.88-0.95)$ \\
Equilibrium (25.8) & 264 & $0.88(0.83-0.92)$ \\
Peak venous (17.7) & 181 & $0.85(0.79-0.91)$ \\
Late venous (7.0) & 72 & $0.76(0.64-0.89)$ \\
Slice thickness $(\%)$ & & \\
$>2$ mm (10.8) & 111 & $0.87(0.81-0.94)$ \\
\hline
\end{tabular}

Dependent variable: dichotomized visual collateral score (0-1: poor collaterals, 2-3: good collaterals). $M C A$, middle cerebral arteries; $A U C$, Area Under the Curve; $\mathrm{mm}$, millimeters. ${ }^{\text {a }}$ Scan phase not detected in 1 patient

visual score which resulted in a Spearman $\rho$ of 0.75 [9]. Another study with 89 patients from Grunwald et al assessed the performance of an automated CTA collateral score algorithm (Brainomix Ltd.), which uses a combination of classical image processing techniques and machine learning classifiers to produce a categorical CS of $0-3$ [8]. The authors reported $90 \%$ agreement and an ICC of 0.93 with a visual collateral score based on consensus between 3 neuroradiologists. However, the neuroradiologists were informed on the automated CTA collateral score [8]. Recently, a study on 269 patients reported the performance of an algorithm using a deep learning convolutional neural network to produce both a categorical CS (0-3) and qCS [7]. The reference standard was produced by two radiologists, and a third radiologist in case of disagreement, who all scored the visual CS independently on baseline CTA imaging. For the categorical CS in comparison to the visual CS, an agreement of $80 \%$ was reported, which increased to $90 \%$ for the dichotomized CS $(0-1,2-3)$ [7]. Lastly, a study with 86 patients published results from ColorViz, a tool to assess collateral circulation during stroke [10]. Two neuroradiologists scored collateral status on multiphase CTA (mCTA) using a 6-point scale [19] which was compared to a 3-point visual scale that scored the automated color-coded summation maps displaying the intracranial vasculature using the mCTA scan. When using this tool, neuroradiological expertise is still obligatory, since no automated score is produced by ColorViz.

Although collateral status is not commonly used in clinical practice in treatment decisions, it is an established treatment effect modifier [4]. Also, collateral status predicts outcome [20]. Therefore, fast, reliable, and consistent collateral scoring is of utmost importance. The predictive value of CS is low with an AUC of 0.64 (vCS) and 0.66 (qCS). However, this is a prediction model with just one parameter. A recently published multivariable model to predict functional independence showed an AUC of 0.73-0.80 in 4 cohorts, but needed 11 baseline clinical and radiological parameters to achieve this [1]. From these parameters, collateral score was the strongest radiological predictor for outcome, even surpassing predictive performance of the occlusion location [2]. This makes the collateral score the excellent candidate for automation in order to facilitate the user with all associated benefits (consistent, always available, visualization of intracranial vessels). This study demonstrates that qCS provides a reliable replacement for visual collateral scoring. Neuroradiological expertise for reliable collateral scoring is not always available in acute stroke, especially during shift hours or in smaller hospitals. Since time is brain -every minute of untreated ischemic stroke, millions of brain cells die-, automated tools that have the potential to expedite stroke treatment may contribute to better outcome. After non-contrast CT, CTA is the most commonly requested imaging in suspected stroke patients, which makes a CTA-based qCS easy to implement. However, this study could not demonstrate a better prediction of outcome with QCS compared to visual score. This is in line with a previous study which reported similar performance of vCS versus qCS [9]. Prediction and prognostication in clinical practice should in the end be performed with a model that contains both clinical and imaging parameters [21].

There are a few limitations to our study. Due to the large sample size, vCS was scored by only one observer per scan. We addressed this issue by a blind review by the first and last authors independently of scans with vCS outliers in comparison to qCS. From the patients with anterior LVO, $26 \%$ of patients were excluded due to absence of usable CTA imaging. This was mainly due to incomplete head coverage (234/
Table 4 Predicting functional independence (90-day mRS 0-2) with collateral score

\begin{tabular}{lll}
\hline Collateral score & AUC $(95 \%$ CI $)$ & $\begin{array}{l}\text { AUC difference with visual } \\
\text { collateral score* }-p\end{array}$ \\
\hline Visual & $0.64(0.61-0.68)$ & N.A. \\
Automated categorized $0-3 * *$ & $0.63(0.60-0.67)$ & 0.61 \\
Automated continuous (affected MCA) & $0.66(0.62-0.69)$ & 0.29 \\
Automated continuous (whole MCA) & $0.66(0.62-0.69)$ & 0.25 \\
\hline
\end{tabular}

$M C A$, middle cerebral arteries; $A U C$, Area Under the Curve. Dependent variable: dichotomized visual collateral score (0-1: poor collaterals, 2-3: good collaterals). *Using the Delong test ** $0: 0 \%, 1:>0 \%-\leq 50 \%, 2:>50 \%<<$ $100 \%, 3: 100 \%$ 
385) which could be solved in future studies by emphasizing importance of displaying the complete brain in patients with suspected ischemic stroke. This not only enables algorithms to process a larger percentage of scans, but also helps physicians minimize the risk of missing a diagnosis in the non-displayed part of the head. A total of 5\% of all anterior LVO patients were excluded because no occlusion side was detected (45/69, supplementary materials, table 2 ) or an incorrect side was appointed as the affected hemisphere (24/69, supplementary materials, table 3). If no occlusion side was detected, qCS was not assessed, but an incorrectly detected affected hemisphere still resulted in a collateral score, which was mainly higher than the reference visual score, since qCS is computed by comparing the affected hemisphere to the unaffected hemisphere. In future versions of the automated collateral scoring algorithm, this could be addressed by retraining with more cases and focus on occlusion side, especially in cases with high CS. A more pragmatic approach would be to make it possible to give the symptomatic side as input to the algorithm. This enables the algorithm to focus on the corresponding hemisphere, which could reduce errors.

Although algorithm performance was independent of scan phase, a suboptimal scan phase could give an erroneous interpretation of the collateral score in an individual. If a CTA scan is acquired in the late venous phase, the amount of contrast visible in the healthy hemisphere could be lower in comparison to the affected hemisphere due to delayed filling of the vasculature [22]. This can result in an apparent high vCS, even when there is a clear deficit visible on CT-perfusion imaging, but delayed filling in the late venous phase is associated with poor baseline collateral status at baseline [22]. An early arterial scan phase could give similar issues due to delayed filling. This emphasizes the importance of scan phase awareness when assessing the collateral status.

The mean processing time to produce $\mathrm{qCS}$ was $5 \mathrm{~min}$. This should be improved to be able to beat a neuroradiologist in providing a collateral score. Nevertheless, 24/7 availability of computer algorithms, the consistent qCS scoring, and the visualization of intracranial vessels are valuable characteristics for physicians treating patients with a suspected stroke.

\section{Conclusions}

This automated collateral score deep learning-based algorithm performs similar to expert radiologists in categorizing patients with a good versus poor collateral score and in predicting functional independence in acute ischemic stroke patients with a large vessel occlusion.

Supplementary Information The online version contains supplementary material available at https://doi.org/10.1007/s00330-022-08627-4.
Acknowledgements The authors thank NICO.LAB (Amsterdam, The Netherlands) and, in particular, Razmara Nizak, Renan Sales Barros, and Merel Boers for providing access to StrokeViewer and their technical assistance throughout the course of this study. We want to thank the MR CLEAN Registry investigators for their contributions.

Funding MR CLEAN Registry has received funding by stichting Toegepast Wetenschappelijk Instituut voor Neuromodulatie (TWIN) and institutional funds: Amsterdam UMC, Erasmus MC, MUMC+.

The current study is executed within the CONTRAST consortium. The CONTRAST consortium is supported by Netherlands Cardiovascular Research Initiative, an initiative of the Dutch Heart Foundation, by the Brain Foundation Netherlands and powered by Health $\sim$ Holland, Top Sector Life Sciences and receives unrestricted funding from Stryker, Penumbra, Medtronic and Cerenovus.

\section{Declarations}

Guarantor The scientific guarantor of this publication is Aad van der Lugt.

Conflict of interest The authors of this manuscript declare relationships with the following companies.

Lennard Wolff: none

Simone M. Uniken Venema: none

Sven P.R. Luijten: none

Jeannette Hofmeijer: none

Jasper M. Martens: none

Marie Louise E. Bernsen:

Pieter Jan van Doormaal: none

Adriaan C.G.M. van Es: none

Diederik W.J. Dippel: none

Wim van Zwam: The Maastricht UMC+ received funds for consultations done by WHZ for Cerenovus and Stryker Neurovascular.

Theo van Walsum: none

Aad van der Lugt: The Erasmus MC received grants for research from Siemens Healthineers, GE Healthcare, and Philips Healthcare.

Statistics and biometry No complex statistical methods were necessary for this paper.

Informed consent Written informed consent was obtained from all subjects (patients) or their legal representatives in this study.

Ethical approval Institutional Review Board approval was obtained.

Study subjects or cohorts overlap Some study subjects or cohorts have been previously reported in other MR CLEAN Registry publications.

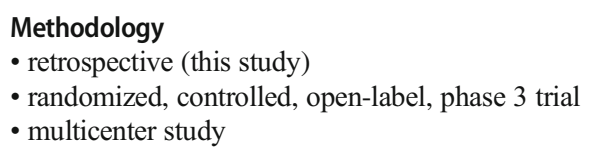

Open Access This article is licensed under a Creative Commons Attribution 4.0 International License, which permits use, sharing, adaptation, distribution and reproduction in any medium or format, as long as you give appropriate credit to the original author(s) and the source, provide a link to the Creative Commons licence, and indicate if changes were made. The images or other third party material in this article are included in the article's Creative Commons licence, unless indicated otherwise in a credit line to the material. If material is not included in the article's 
Creative Commons licence and your intended use is not permitted by statutory regulation or exceeds the permitted use, you will need to obtain permission directly from the copyright holder. To view a copy of this licence, visit http://creativecommons.org/licenses/by/4.0/.

\section{References}

1. Goyal M, Menon BK, van Zwam WH et al (2016) Endovascular thrombectomy after large-vessel ischaemic stroke: a meta-analysis of individual patient data from five randomised trials. Lancet 387 : 1723-1731

2. Venema E, Mulder M, Roozenbeek B et al (2017) Selection of patients for intra-arterial treatment for acute ischaemic stroke: development and validation of a clinical decision tool in two randomised trials. BMJ 357:j1710

3. Jansen IG, Mulder MJ, Goldhoorn RB et al (2019) Impact of single phase CT angiography collateral status on functional outcome over time: results from the MR CLEAN Registry. J Neurointerv Surg. https://doi.org/10.1136/ neurintsurg-2018-014619

4. Berkhemer OA, Jansen IG, Beumer D et al (2016) Collateral status on baseline computed tomographic angiography and intra-arterial treatment effect in patients with proximal anterior circulation stroke. Stroke 47:768-776

5. Tan IY, Demchuk AM, Hopyan J et al (2009) CT angiography clot burden score and collateral score: correlation with clinical and radiologic outcomes in acute middle cerebral artery infarct. AJNR Am J Neuroradiol 30:525-531

6. McVerry F, Liebeskind DS, Muir KW (2012) Systematic review of methods for assessing leptomeningeal collateral flow. AJNR Am J Neuroradiol 33:576-582

7. Su J, Wolff L, van Es A et al (2020) Automatic collateral scoring from 3D CTA images. IEEE Trans Med Imaging 39:2190-2200

8. Grunwald IQ, Kulikovski J, Reith W et al (2019) Collateral automation for triage in stroke: evaluating automated scoring of collaterals in acute stroke on computed tomography scans. Cerebrovasc Dis 47:217-222

9. Boers AMM, Sales Barros R, Jansen IGH et al (2018) Value of quantitative collateral scoring on $\mathrm{CT}$ angiography in patients with acute ischemic stroke. AJNR Am J Neuroradiol 39:1074-1082

10. Verdolotti T, Pilato F, Cottonaro S et al (2020) ColorViz, a new and rapid tool for assessing collateral circulation during stroke. Brain Sci. https://doi.org/10.3390/brainsci10110882

11. Aktar M, Tampieri D, Rivaz H, Kersten-Oertel M, Xiao Y (2020) Automatic collateral circulation scoring in ischemic stroke using
4D CT angiography with low-rank and sparse matrix decomposition. Int J Comput Assist Radiol Surg 15:1501-1511

12. Jansen IGH, Mulder M, Goldhoorn RB, MR CLEAN Registry investigators (2018) Endovascular treatment for acute ischaemic stroke in routine clinical practice: prospective, observational cohort study (MR CLEAN Registry). BMJ 360:k949

13. Bossuyt PM, Reitsma JB, Bruns DE et al (2015) STARD 2015: an updated list of essential items for reporting diagnostic accuracy studies. Radiology 277:826-832

14. Boers AMM Sales Barros R, Jansen IG, Slump CH et al (2017) Quantitative collateral grading on CT angiography in patients with acute ischemic stroke. In: Cardoso $\mathrm{M}$ et al (eds) Molecular Imaging, Reconstruction and Analysis of Moving Body Organs, and Stroke Imaging and Treatment. RAMBO 2017, CMMI 2017, SWITCH 2017. Lecture Notes in Computer Science, vol 10555. Springer, Cham https://doi.org/10.1007/978-3-319-67564-0 18

15. Luijten SPR, Wolff L, Duvekot MHC et al (2021) Diagnostic performance of an algorithm for automated large vessel occlusion detection on CT angiography. J Neurointervent Surg. https://doi.org/ 10.1136/neurintsurg-2021-017842

16. Rodriguez-Luna D, Dowlatshahi D, Aviv RI et al (2014) Venous phase of computed tomography angiography increases spot sign detection, but intracerebral hemorrhage expansion is greater in spot signs detected in arterial phase. Stroke 45:734-739

17. Koo TK, Li MY (2016) A guideline of selecting and reporting intraclass correlation coefficients for reliability research. J Chiropr Med 15:155-163

18. DeLong ER, DeLong DM, Clarke-Pearson DL (1988) Comparing the areas under two or more correlated receiver operating characteristic curves: a nonparametric approach. Biometrics 44:837-845

19. Menon BK, d'Esterre CD, Qazi EM et al (2015) Multiphase CT angiography: a new tool for the imaging triage of patients with acute ischemic stroke. Radiology 275:510-520

20. Maas MB, Lev MH, Ay $\mathrm{H}$ et al (2009) Collateral vessels on CT angiography predict outcome in acute ischemic stroke. Stroke 40: 3001-3005

21. Venema E, Roozenbeek B, Mulder M et al (2021) Prediction of outcome and endovascular treatment benefit: validation and update of the MR PREDICTS Decision Tool. Stroke 52:2764-2772

22. Bhaskar S, Bivard A, Parsons M et al (2017) Delay of late-venous phase cortical vein filling in acute ischemic stroke patients: associations with collateral status. J Cereb Blood Flow Metab 37:671682

Publisher's note Springer Nature remains neutral with regard to jurisdictional claims in published maps and institutional affiliations. 